\title{
On the theory of reactive mixtures for modeling biological growth
}

\author{
Gerard A. Ateshian
}

Published online: 25 July 2007

(C) Springer-Verlag 2007

\section{Erratum to: Biomechan Model Mechanobiol}

\section{DOI 10.1007/s10237-006-0070-x}

An error in sign is corrected in the jump condition on the entropy,

$$
\begin{aligned}
& \sum_{\alpha}\left[\left[\rho^{\alpha} \eta^{\alpha} \mathbf{u}_{\Gamma}^{\alpha}+\frac{\mathbf{q}^{\alpha}}{\theta}\right]\right] \cdot \mathbf{n} \leq 0 \\
& {\left[\left[\rho \eta \mathbf{u}_{\Gamma}+\frac{\mathbf{h}}{\theta}\right]\right] \cdot \mathbf{n} \leq 0}
\end{aligned}
$$

which also requires the following sign corrections,

$$
\begin{aligned}
& {\left[\left[\rho^{\alpha}\left(\varepsilon^{\alpha}+\frac{1}{2} \mathbf{u}_{\Gamma}^{\alpha} \cdot \mathbf{u}_{\Gamma}^{\alpha}\right) \mathbf{u}_{\Gamma}^{\alpha}-\left(\mathbf{T}^{\alpha}\right)^{T} \mathbf{u}_{\Gamma}^{\alpha}+\mathbf{q}^{\alpha}\right]\right] \cdot \mathbf{n}=-\bar{\varepsilon}^{\alpha}} \\
& {\left[\left[\rho^{\alpha} \theta \eta^{\alpha} \mathbf{u}_{\Gamma}^{\alpha}+\mathbf{q}^{\alpha}\right]\right] \cdot \mathbf{n}=-\theta \bar{\eta}^{\alpha}} \\
& {\left[\left[\rho^{\alpha}\left(\mathbf{K}^{\alpha}+\frac{1}{2}\left(\mathbf{u}_{\Gamma}^{\alpha} \cdot \mathbf{u}_{\Gamma}^{\alpha}\right) \mathbf{I}\right) \mathbf{u}_{\Gamma}^{\alpha}\right]\right] \cdot \mathbf{n}=-\bar{\psi}^{\alpha}}
\end{aligned}
$$

The online version of the original article can be found under doi:10.1007/s10237-006-0070-x.

\section{G. A. Ateshian $(\varangle)$}

Department of Mechanical Engineering,

Columbia University,

500 West 120th St., MC4703,

220 S.W. Mudd, New York,

NY 10027, USA

e-mail: ateshian@columbia.edu 